\title{
POWER OPTIMIZATION OF MULTI-FLUID TRANSPORTATION SYSTEMS
}

\author{
E. F. Camacho, M. A. Ridao and J. A. Ternero \\ Departamento de Automática, E.T.S.I.Industriales, Sevilla, Spain
}

\begin{abstract}
This paper presents an algorithm for optimizing the energy operating costs in multi-fluid transportation systems. The algorithm works in two steps. The first one consists of the computation of a function that measures the estimated minimum cost to the goal node. This computation involves the use of Bellman's optimality principle and some heuristic rules in order to avoid the combinatorial explosion. During the second step the optimum trajectory is obtained with the help of the function mentioned above and using an accurate simulation of the transportation system. The algorithm is applied to a model of an oil pipeline system.
\end{abstract}

Keywords. Optimization; multifluid transportation systems; dynamic programming.

\section{INTRODUCTION}

Multi-fluid transportation systems consist of a pipeline network, a set of valves, pumps and storage tanks. The products are transported through the pipeline network from the input nodes to the terminal nodes where there are normally stored in tanks. The product is then extracted from the storage tanks by the consumers in a continous or semi continous way. The fluid has to be transported, taking into account the system's capabilities, in order to satisfy the consumers' needs, that is, making sure that there is always enough products in the storage tanks, that the tanks at each terminal can receive the product delivered at any given moment and that the product can be delivered throughout the system as required.

The energy optimization problem consists in minimizing the power bill while satisfying the restrictions mentioned above. The interest of such an optimization is evident as power costs are increasing in respect to the total operating costs of fluid transportation systems as shown in (Basavaraj, 1984).
In order to obtain a realistic solution to the optimization problem, the following should be taken into account:

- Topology and topography of the network.

- Non-linear characteristics of pumps and pipelines.

- Variable demands of consumers.

- Time changing prices of electrical energy.

- Hydraulic equations throughout the systems.

The problem has been dealt with in the literature, especially for mono-fluid transportation systems, as is the case of water distribution systems (Coulbeck, 1978), (Millaoka et al. 1984). However, even in this case, the optimization problem leads to a dynamic and non-linear problem of high dimension as stated in (Kemplous et al. 1986). In this last reference an optimization algorithm that works at two levels has been proposed. At the upper level the dynamic problem is solved and some data passed to the lower level where the static problem is solved giving the number of pumps turnned-on in the pumping station. 
The qualitative and quantitative complexity of the problem is substantially increased by taking into account multi-fluid capabilities. This problem has been dealt with in literature, in (Burham, 1983) the potencial savings of an adequate operation are shown.

This article presents an algorithm for power cost optimization in multi-fluid transportation systems. The algorithm is basically a dynamic programming algorithm and works in two steps. In the first step a function that measures the optimum expected cost to the goal node is computed by the use of some simplifying assumptions. In the second step, the optimum trajectory is determined by taking into account the previously mentioned function and using a more accurate simulation of the transportation system. The algorithm has been applied to different oil pipeline models.

The paper is organized as follows: Section 2 describes the way in which multi-fluid transportation systems are operated and how the optimization problem is formulated. Section 3 is devoted to the optimization algorithm while an application of the algorithm to an oil pipeline is presented in section 4. Finally the conclusions are presented in section 5 .

\section{PROBLEM DESCRIPTION}

In multi-fluid transportation systems, the different fluids are injected into the system at input nodes and then transported to the destination nodes through the pipeline network. At any given time different products ( separated by the corresponding interfaces ) can be transported. The products are routed through the network by opening or closing the appropiate set of valves.

The mechanical energy needed for the transportation is supplied by a set of electrically powered pumps. The transportation velocity for a particular network and a given pipeline state, depends on the number and the flow-head characteristics of the pumps working at each of the pumping stations.

The operation of a multi-fluid transportation system consists in determining the order and the way in which the different fluids are going to be transported to satisfy the demand. This task can be divided into two different problems. The first one involves the determination of the ap- proximate transportation needs. The solution of this problem will consist in

- a batch sequence that will minimize the number of interfaces and will cover the consumption needs at each destination node.

- an approximate pumping schedule.

The batch sequence consists of a list of batches each of which is defined by the product identification, approximate departure time, the volume destined to each output node and approximate arrival time to each terminal node.

The second problem involves determining how to set the different pumps and valves at each time interval in such a way that the batch sequence given above is carried out in a given period of time. This can be accomplished in different ways, that is, using different pump combinations and valve positions ( and therefore flows at all points in the network ) in the different time intervals. This will turn into different batch arrival times, and different power operating costs as the price of electricity changes throughout the day and also friction losses and pump performances depend on the flow.

The time intervals mentioned above are defined by certain events. These are produced when significant changes occur within the system. The main events are:

- Changes in electrical tariffs.

- Batch arrival to nodes.

- Tank levels reaching limits.

- Shut-down of the system.

- Periodic events.

The periodic events are introduced to guarantee that the time interval between two consecutive events is small. In this case, the pipeline state does not change substancially from one event to the next, and the pipeline state can be considered constant between two consecutive states. Computations are greatly simplified this way.

The power cost optimization problem consists in finding out which way of operating the system (that is. how to set the pumps and valves at each time interval) is the cheapest and fulfils the needs mentioned above. 
In the following it will be consider that the system is operated in the "stripping" mode. That is, each output node is working with an input flow proportional to the amount of product that the terminal has to receive from the current batch. This method of operating is widely considered as the best way of using the transportation capabilities of the pipelines.

Notice that if the stripping mode is used, the amount of product stripped at each terminal depends on the batch passing in that moment. Therefore, for a given pipeline state the flow at each terminal, and at each bifurcation node, can be computed knowing the flow at the entry node. The pipeline state can be computed from the volume injected into the entry node and the batch sequence.

When operating in the stripping mode the future evolution of the system can be computed if the following items are known.

- amount of product which has been pumped (v)

- the time used $(\mathrm{t})$

- the setting of the pumps

- the flow at the entry node.

The state of the system can, therefore, be resumed by the pair $(v, t)$. The control variables are the pump setting and the flow at the entry point. By looking at typical characteristics of pump power consumption - flow, it is clear that the power consumption of the pumps grows slowly with the flow and depends mainly on the pump being connected or not. Therefore, from the power optimization point of view, the best results will be obtained when the maximum flow is chosen.

Taking into account these considerations, the control variables will be a vector of dimension equal to the number of pumps in the system containing a logical value which will indicate if the corresponding pump is active. The number of combinations of the previously mentioned vector can be reduced for pumping stations with identical pumps. In this last case, knowing the number of pumps connected ( in series or parallel) is enough.

The working space will be the state plane $\mathrm{v}$-t and the optimum control problem will consist in determining an optimum trajectory in the plane $\mathrm{v}$ - $\mathrm{t}$ from point $(0,0)$ to point (target-volume,targettime).

The feasible trajectories are constrained by two curves as indicated in figure 1. The upper one is the curve resulting when pumping the batch sequence using the maximum capabilities of the system. Notice the horizontal pieces of the curve corresponding to intervals where the pumps have

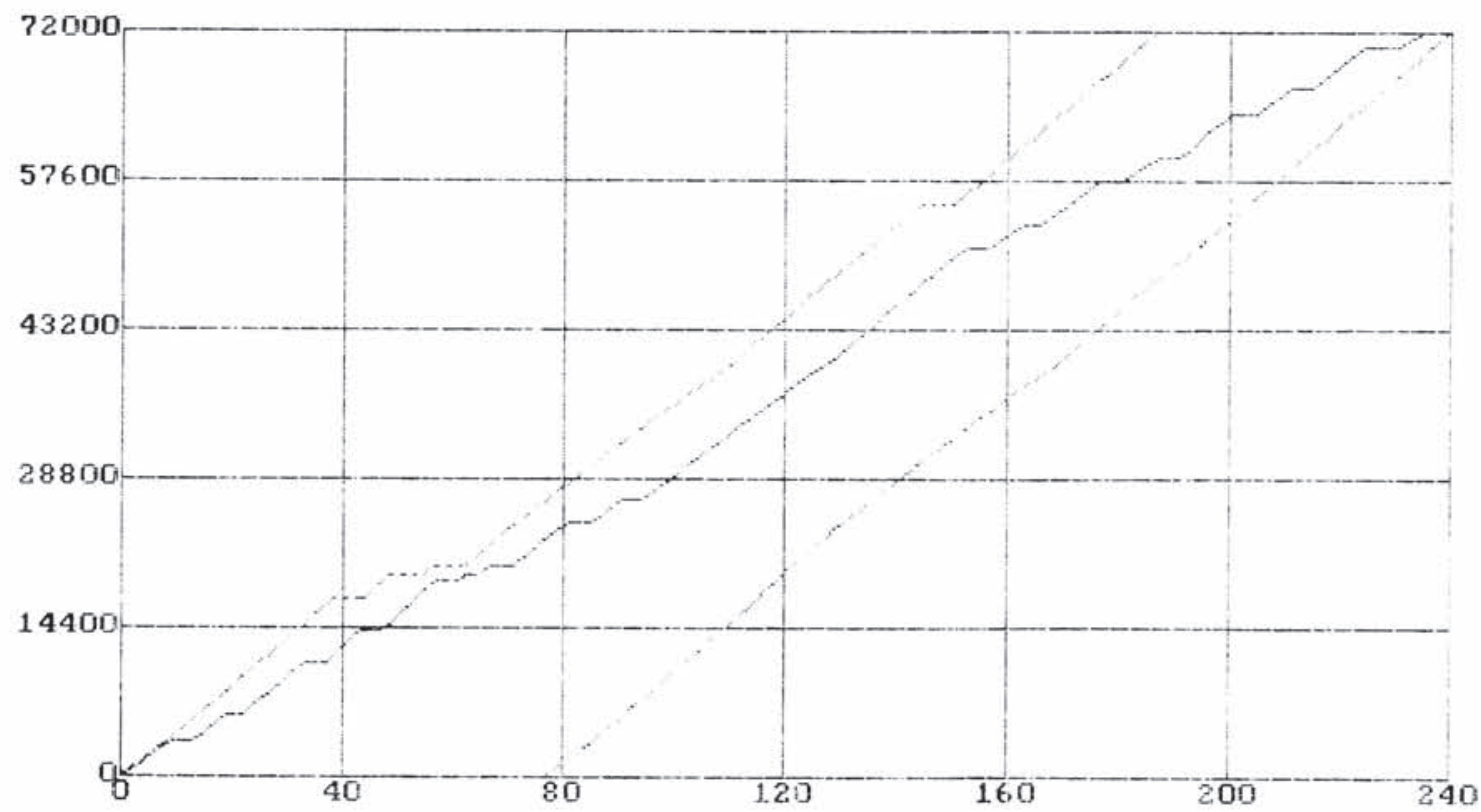

Fig. 1. Feasible Optimization Space State. 
to stop either to avoid tank overflow or due to maintenance shut-down of the system. The lower curve is computed taking into account the minimum pumped amount at each interval in order to satisfy the needs and taking into account the system pumping capabilities.

\section{OPTIMIZATION ALGORITHM}

The optimization algorithm uses the cost function:

$$
f\left(x_{v, t}\right)=g\left(x_{v, t}\right)+h\left(x_{v, t}\right)
$$

where $x_{v, t}$ is the state vector and consists of a pair (volume,time), $g\left(x_{v, t}\right)$ is a function representing the minimum cost from the origin to node $x_{v, t}$ and $h\left(x_{v, t}\right)$ is a function that estimates the minimum cost from $x_{v, t}$ to a goal node. That is, $f\left(x_{v, t}\right)$ is an estimate of the cost of the minimal cost path constrained to go through node $x_{v, t}$.

The optimization algorithm works in two steps. In the first one the function $h\left(x_{v, t}\right)$ is computed on a grid by using Bellman's Optimality Principle and in the second one the optimum path is found by using this function and an accurate simulation of the transportation system.

In the first step of the algorithm, function $h\left(x_{v, t}\right)$ is made equal to zero for all $x_{v, t}$ with volume equal or greater than the target volume. The next step for estimating the function $h\left(x_{v, t}\right)$ is to compute all states $x_{v_{1}, t_{1}}$ from where $x_{r, t}$ can be reached. Notice that if the volume difference $\Delta v=v-v_{1}$ is small, the pipe state will be practically the same. Therefore. for a given pump combination the flow will be very similar and can be considered constant.

The time $t_{1}$ for each pump combination can be determined by the intersection of the line $\mathrm{L} 1$, see figure 2, that goes through state $x_{v, t}$ with a slope equal to the flow for the corresponding pumping combination and the horizontal line L2 of volume equal to $v_{1}$. If the line $\mathrm{L} 1$ crosses a shut down period the computation of time $t_{1}$ should be made as indicated in figure 2 .

In order to avoid the combinatorial explosion, a procedure for eliminating states is used. At first, the procedure eliminates the trivial states, and then a set of heuristic rules are used to decicle which states are "the best" for the next step of the algorithm.

A state $x_{v, t}$ is considered to be trivial if there is another state $x_{v, t_{i}}$ with

$t_{i} \geq t$ and

$h\left(x_{v, t}\right) \geq h\left(x_{v, t_{i}}\right)$.

Notice that state $x_{v, t}$ could be reached from $x_{v, t_{i}}$ by switching off all the pumps between $t_{i}$ and $t$ (with cost zero) and the function $h\left(x_{v, t}\right)$ will therefore be equal to $h\left(x_{v, t_{i}}\right)$.

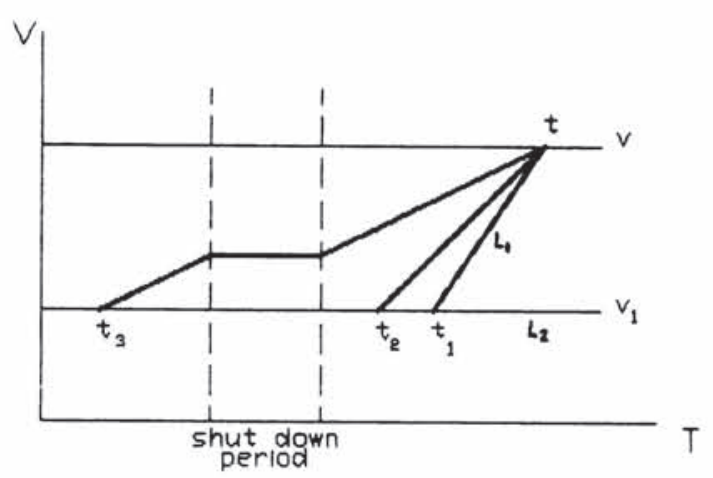

Fig. 2. State Transitions.

Once all trivial state have been eliminated, a second elimination procedure takes place in order to obtain a feasible amount of states for the line L2. The segment of L2 including the limit curves (figure 1) is divided into a number of regions. At each region the point with "the best" characteristics is chosen. This guarantiees that the function $h\left(x_{v, t}\right)$ is computed over a grid covering the feasible space.

Once the function $h\left(x_{v, t}\right)$ has been computed over the grid, the second step of the algorithm can be carried out. It consists of computing, at each interval and for all possible pumping combinations, the accumulated cost to the next event $g\left(x_{v, t}\right)$ plus the estimated cost to the goal node $h\left(x_{v, t}\right)$. The function $h\left(x_{v, t}\right)$ is calculated by a linear interpolation in the grid computed in the first part of the algorithm.

The accumulated cost is computed by using an accurate simulation of the pipeline network. It involves the computation of pressure drops, flow, power consumption etc. and it takes into account the non-linear characteristics of pumps and pipelines as well as different electricity tariffs throughout the day. 


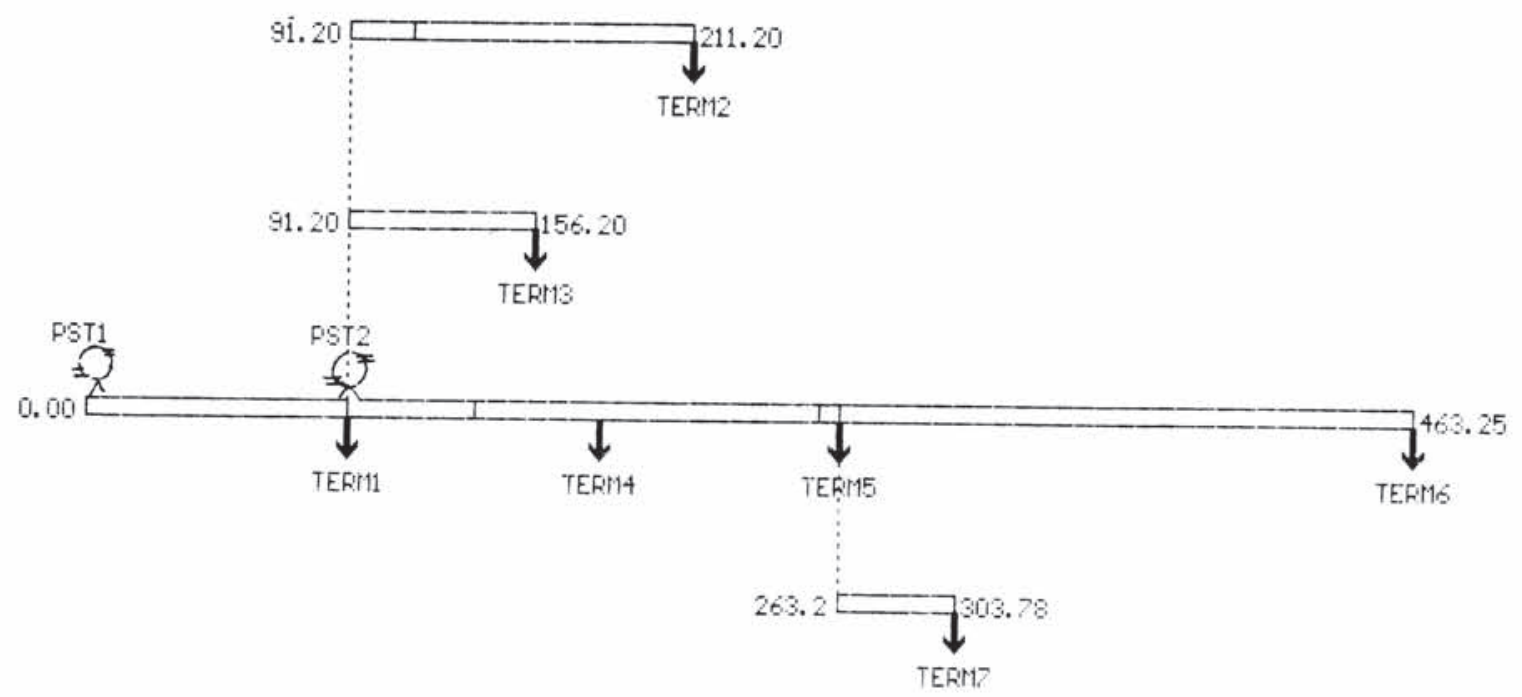

Fig. 3. Pipeline Model.

\section{APPLICATION TO OIL PIPELINES}

The optimization algorithm has been included in a program developed for CAMPSA. The program can be applied to any pipeline transportation system with only one entry node. The algorithm has been tested with the model of an oil pipeline shown in figure 3 .

The pipeline has seven terminals and two pump stations. The first one has three booster pumps operating in parallel and four main pumps operating in series. The second one has three main pumps operating in series. The three booster pumps have ideutical characteristics, and so do the main pumps at the first pumping station. The three main pumps at the second pumping station are also identical. The pipeline has a main pipe and three branches. Two of them leave the main pipe at $\lim 91$ and the other one at $\lim 263$.

Five types of products, two types of gasoline, two types of diesel oil and kerosene, were considered.

The algorithm has been tested with different cases. Table I shows one of the batch sequences used in the tests. Figure 1 corresponds to the solution in a case covering the batch schedule during ten days. Notice that the upper curve has a few horizontal pieces corresponding to points where the

TABLE 1 Batch Sequence.

\begin{tabular}{|c|c|c|c|c|c|c|c|c|}
\hline MAME & VOLUHEN $\left(H^{3}\right)$ & TERMI $(x)$ & IERM2 $(x)$ & TERM $3(x)$ & PERM4 $(x)$ & TERKS $(x)$ & TERNG $(x)$ & IERM7 $(x)$ \\
\hline $\mathrm{P1} / \mathrm{O} 18 / \mathrm{GOC}$ & 10000.00 & 0.00 & 20.00 & 35.80 & 5.00 & 8.08 & 8.08 & 48.00 \\
\hline $\mathrm{P} 1 / 013 / 698$ & 7800.68 & 10.80 & 40.00 & 0.80 & 0.00 & 0.80 & 58.80 & 0.00 \\
\hline $\mathrm{P} 1 / 019 / \mathrm{GOC}$ & 9800.00 & 8.00 & 38.00 & 20.60 & 0.80 & 0.80 & 40.00 & 10.00 \\
\hline $\mathrm{P1} / 026 / 60 \mathrm{~A}$ & 6800.08 & 8.00 & 0.80 & 8.80 & 0.00 & 66.67 & 0.80 & 33.33 \\
\hline P1/014/698 & 15800.80 & 0.00 & 0.00 & 38.08 & 0.00 & 0.00 & 48.00 & 38.08 \\
\hline P1/804/096 & 8000.00 & 0.80 & 0.00 & 0.00 & 25.00 & 8.00 & 0.60 & 75.08 \\
\hline
\end{tabular}


TABLE 2 Optimal Solutions for Different Cases.

\begin{tabular}{|c|c|c|c|c|}
\hline IMITIAL DATE & TARGEI TIME (DAYS) & IARGET VOLUAE $\left(n^{3}\right)$ & MOH-ZARIFFS & COSI (PTS.) \\
\hline $0: 00 \quad 1 / 8 / 89$ & $?$ & 55800 & 2 & 1.235 .318 \\
\hline $8: 80 \quad 1 / 8 / 89$ & 18 & 55800 & 2 & 1.066 .495 \\
\hline $0: 00 \quad 1 / 8 / 89$ & 15 & 55008 & 5 & 940.510 \\
\hline$\theta: \theta 8$ 11/8/89 & ? & 55800 & 3 & 1.111 .791 \\
\hline
\end{tabular}

tanks would overflow if the pumping continued at maximum flow.

The different slopes in the curves correspond to different flows due to different pumping conditions as fluids in the pipeline and flows in the output terminals change with state.

The lower curve corresponds, as mentioned before, to the minimum volume that has to be pumped at each time interval in order to satisfy the consumers' needs. Notice that the pipeline is kept switch off and that the maximun flow is used in order to pump the target volume in the prescribed time.

The thicker line corresponds to the optimum trajectory. It can be seen that the solution involves shutting down all pumps during peak hours, and using high flow combinations during valley periods.

Good economical results have been achieved using the algorithm in the management of the oil pipeline. Table II shows the different solutions obtained when using different target times. As the target time increases the products can be transported during off-peak hours and the cost therefore decreases.

The energy cost has been reduced in approximately 20 per cent from an initial solution.

\section{CONCLUSIONS}

An algorithm for the power cost optimization in multi-fluid transportation systems has been presented. The algorithm uses a function that measures the estimated minimum cost to the goal node and an accurate simulation of the trans- portation system. The algorithm has been tested with success in an oil pipeline model.

\section{ACKNOWLEDGEMENT}

The authors would like to acknowledge the finantial support of CAMPSA for this work and also to thank S. Heras, J.M Rodriguez and R. LLorente of the Departamento de Oleoductos y Operaciones of CAMPSA for their valuable comments. Further thanks is due to F. J. Vidal, C. del Valle, J. Gómez and R. Millán for their collaboration.

\section{REFERENCES}

Basavaraj. B.H. (1984) Cut pipeline power cost by demand control. Oil \& Gas Journal, Jan, pp. 88-90.

Burham C.G. (1983) Rate Schedule Application - Off Peak Operation of Pump Station. Proc. of the Pipeline Engineering Symposium, pp. 11-16, Houston, Texas.

Coulbeck B. and M. Sterling. (1978) Optimizated Control of Water Distribution Systems. Proc. IEEE, Vol. 122, N. 2.

Iilempous R, J. Kotwosky, J. Nikodem and J. Ulasiewicz. (1986), Water Distribution Systems. Components, Instruments and Techniques for Low Cost Automation \& Applications, pp. 541-551, Valencia (Spain).

Miyaoka S., M. Funabashi. (1984) Optimal Control of Water Distribution Systems by Network Flow Theory". IEEE Transaction on Automatic Control, Vol. AC-29, N. 4, pp. 303-311. 